\title{
On the Determination of Velocities of Radar Meteors
}

\author{
P. Pecina \\ Astronomical Institute of the Czech Academy of Sciences, \\ 25165 Ondřejov, Czech Republic, e-mail: ppecina@asu.cas.cz
}

\begin{abstract}
The results of testing the classical method of velocity determination based on the positions of the extrema of Fresnel diffraction patterns (FDP) in radio echo amplitude, is presented. This technique is compared to the method of nonlinear least squares fitting (NLSF) of the theoretical patterns to the observed those. It is shown that while the classical method can fail in yielding correct values for the velocity of radar meteors, the NLSF method always yields good results.
\end{abstract}

\section{Introduction}

One method commonly used to determine the velocity of radar meteors is based on the comparison of the positions of the extrema of the theoretical FDP with those of the observed pattern. The classical FDP method is briefly described in the next section. To the author's knowledge, nobody has yet tried to check this technique's ability to correctly determine the velocity of radar meteors. To test the classical method, we decided to generate 100 theoretical FDP with known starting parameters (see the description of the classical method). These were chosen randomly from within the following intervals: the velocity from 10 to 70 $\mathrm{km} \mathrm{s}^{-1}$, the range from 100 to $300 \mathrm{~km}$, the normalizing factor from 20 to 60 , the time of passage through the specular point from $0.01 \mathrm{~s}$ to $0.02 \mathrm{~s}$, and the ambipolar diffusion coefficient from 0 to $10 \mathrm{~m}^{2} \mathrm{~s}^{-1}$. For purposes of illustration the wavelength was set to $11.45 \mathrm{~m}$ and the pulse repetition frequency to $379 \mathrm{~Hz}$. Theoretical amplitudes were stored with an accuracy of 3 digits. The method of velocity determination relying on the positions of extrema of FDP, was then applied to generated FDP. To compare to these results, the method of NLSF (e.g. Pecina 1988) of theoretical amplitudes to those generated was also applied. The results given by both methods are summarized in Table 1.

\section{Brief description of classical method}

The FDP including diffusion are described by the formula (e.g. Simek 1968)

$$
A_{i}=c_{n} \sqrt{S_{i}^{2}+C_{i}^{2}}
$$

where $A_{i}$ represents the $i$-th amplitude oscillation of the FDP, $c_{n}$ the corresponding normalizing factor, and

$$
S_{i}=\int_{-\infty}^{x_{i}} \exp \left[-a\left(x_{i}-x\right)\right] \sin \left(\pi x^{2} / 2\right) d x, C_{i}=\int_{-\infty}^{x_{i}} \exp \left[-a\left(x_{i}-x\right)\right] \cos \left(\pi x^{2} / 2\right) d x,
$$

where $x_{i}=2 v\left(t_{i}-t_{0}\right) / \sqrt{\lambda R_{0}}$ and $a=8 \pi^{2} D \sqrt{\lambda R_{0}} / v \lambda^{2}$. In the preceding expressions, $v$ designates meteoroid velocity, $D$ stands for the ambipolar diffusion coefficient, $R_{0}$ represents the range to the meteor trail at the specular point, and $\lambda$ is the wavelength of wave transmitted by the radar. The time variable corresponding to $A_{i}$ is designated as $t_{i}$, and the time of meteoroid passage through the specular point as $t_{0}$. Both $S_{i}$ and $C_{i}$ can be evaluated analytically. 
As already stated, the classical method utilizing the FDP for velocity determination compares theoretical extrema positions with those observed. Equating the derivative with respect to $x_{i}$ of eq.(1) to zero we arrive at the equation

$$
S_{e i} \sin \left(\pi x_{e i}^{2} / 2\right)+C_{e i} \cos \left(\pi x_{e i}^{2} / 2\right)-a\left(S_{e i}^{2}+C_{e i}^{2}\right)=0,
$$

containing the extrema position variable, $x_{e i}$, and also $a$ as unknowns. The following iteration process was shown to work. First, we assume $a=0$. We can then compute $x_{e i}$ from eq.(3). Second, from the ratio of two known amplitudes in the extrema, we get equation

$$
A_{e i} \sqrt{S_{e j}^{2}+C_{e j}^{2}}=A_{e j} \sqrt{S_{e i}^{2}+C_{e i}^{2}}
$$

and a new value of $a$ is obtained. Returning back to eq.(3) we get more precise values of $x_{e i}$. The iteration process can be terminated if two subsequent values of the parameters in question differ by less than some prescribed accuracy. This process converges rapidly, generally only a few iterations are needed in most cases. For our computations we used the ratio of the first maximum to the second minimum. Then the velocity of the meteoroid can be evaluated from the formula

$$
v=\left(\sqrt{\lambda R_{0}} / 2\right)\left(x_{e j}-x_{e i}\right) /\left(t_{e j}-t_{e i}\right) .
$$

We used the positions of the first two maxima and minima because these are usually well defined in the observational record. Thus, we have six possible combinations to use in deriving $v$ from eq. (5), as indicated in Table 1 . Here $\bar{v}$ was computed from these six quantities while $v_{l s}$ was computed by the NLSF method for which special attention was paid to finding the initial values of parameters entering iteration process.

Table 1. Input velocities, $v_{i}$, of generated FDP, velocities resulting from $i$-th and $j$-th extreme, $v_{i-j}$, their average, $\bar{v}$, and the velocity, $v_{l s}$, resulting from the application of the NLSF method to generated FDP

\begin{tabular}{r|c||cccccc||c|c} 
No. & $v_{i}$ & $v_{1-2}$ & $v_{1-3}$ & $v_{1-4}$ & $v_{2-3}$ & $v_{2-4}$ & $v_{3-4}$ & $\bar{v}$ & $v_{l s}$ \\
\hline 1 & 13.421 & 13.220 & 13.243 & 13.278 & 13.291 & 13.337 & 13.377 & 13.291 & 13.421 \\
2 & 35.791 & 39.711 & 36.756 & 37.456 & 33.063 & 35.846 & 39.556 & 37.065 & 35.791 \\
3 & 66.779 & 90.349 & 77.087 & 69.496 & 63.824 & 59.069 & 54.315 & 69.023 & 66.784 \\
4 & 24.101 & 25.584 & 25.541 & 24.057 & 25.465 & 22.869 & 20.791 & 24.051 & 24.103 \\
5 & 29.971 & 30.454 & 30.904 & 29.906 & 31.805 & 29.420 & 27.512 & 30.000 & 29.976 \\
6 & 54.846 & 58.911 & 53.746 & 57.173 & 46.859 & 55.782 & 69.167 & 56.940 & 54.840 \\
7 & 13.967 & 13.931 & 13.872 & 13.963 & 13.744 & 13.996 & 14.225 & 13.955 & 13.968 \\
8 & 46.901 & 55.401 & 44.597 & 45.698 & 33.794 & 39.877 & 49.002 & 44.728 & 46.910 \\
9 & 65.829 & 54.966 & 68.180 & 30.920 & 107.821 & 22.905 & 12.290 & 49.514 & 65.832 \\
10 & 34.325 & 35.274 & 35.825 & 34.662 & 36.653 & 34.203 & 31.754 & 34.728 & 34.324 \\
11 & 65.654 & 83.584 & 71.891 & 64.701 & 60.198 & 55.259 & 50.320 & 64.325 & 65.651 \\
12 & 30.295 & 30.709 & 29.299 & 29.502 & 26.762 & 28.415 & 30.068 & 29.126 & 30.296 \\
13 & 61.909 & 59.945 & 74.429 & 67.496 & 117.880 & 75.048 & 53.631 & 74.738 & 61.913 \\
14 & 38.907 & 35.952 & 24.703 & 36.576 & 33.036 & 37.075 & 43.132 & 36.746 & 38.902 \\
15 & 15.533 & 15.438 & 15.613 & 15.310 & 16.029 & 15.175 & 14.492 & 15.343 & 15.533 \\
16 & 47.622 & 47.154 & 47.588 & 47.266 & 48.311 & 47.359 & 46.408 & 47.348 & 47.620 \\
17 & 60.706 & 70.801 & 69.738 & 59.375 & 68.142 & 52.520 & 42.104 & 60.447 & 60.709 \\
18 & 22.679 & 23.814 & 22.995 & 23.203 & 21.521 & 22.653 & 23.786 & 22.995 & 22.680 \\
19 & 15.919 & 15.024 & 15.393 & 15.398 & 16.192 & 15.771 & 15.410 & 15.531 & 15.919 \\
20 & 16.217 & 15.770 & 15.943 & 16.566 & 16.238 & 17.301 & 18.542 & 16.727 & 16.219 \\
21 & 56.143 & 70.498 & 57.240 & 58.582 & 43.982 & 51.432 & 62.607 & 57.390 & 56.145 \\
22 & 56.677 & 48.901 & 47.391 & 26.967 & 45.127 & 19.655 & 12.377 & 33.403 & 56.687 \\
23 & 13.405 & 13.471 & 13.430 & 13.566 & 13.355 & 13.651 & 13.948 & 13.570 & 13.408 \\
24 & 63.962 & 60.967 & 57.181 & 60.586 & 52.132 & 60.282 & 72.507 & 60.609 & 63.963 \\
25 & 18.948 & 17.732 & 18.635 & 18.197 & 20.079 & 18.569 & 17.059 & 18.378 & 18.944
\end{tabular}


The first continuation of Table 1 .

\begin{tabular}{|c|c|c|c|c|c|c|c|c|c|}
\hline No. & $v_{i}$ & $v_{1-2}$ & $v_{1-3}$ & $v_{1-4}$ & $v_{2-3}$ & $v_{2-4}$ & $v_{3-4}$ & $\bar{v}$ & $v_{l s}$ \\
\hline 26 & 68.147 & 83.988 & 70.417 & 38.224 & 56.845 & 26.873 & 16.762 & 48.836 & 68.140 \\
\hline 27 & 47.989 & 37.075 & 41.530 & 42.253 & 50.442 & 47.431 & 44.420 & 43.858 & 47.986 \\
\hline 28 & 42.530 & 47.972 & 45.494 & 43.300 & 42.189 & 40.185 & 38.181 & 42.887 & 42.532 \\
\hline 29 & 40.025 & 38.495 & 37.452 & 39.410 & 36.063 & 40.143 & 46.262 & 39.638 & 40.019 \\
\hline 30 & 49.701 & 43.541 & 44.079 & 48.137 & 44.975 & 52.734 & 64.372 & 49.640 & 49.704 \\
\hline 31 & 49.073 & 41.203 & 48.461 & 48.287 & 70.234 & 56.787 & 47.822 & 52.132 & 49.074 \\
\hline 32 & 46.534 & 36.871 & 41.116 & 41.868 & 49.606 & 46.864 & 44.123 & 43.408 & 46.533 \\
\hline 33 & 52.455 & 41.504 & 47.210 & 47.882 & 58.620 & 54.260 & 49.900 & 49.896 & 52.454 \\
\hline 34 & 55.311 & 51.204 & 57.996 & 55.566 & 74.977 & 59.929 & 49.897 & 58.262 & 55.315 \\
\hline 35 & 46.356 & 39.665 & 44.171 & 44.990 & 53.184 & 50.316 & 47.447 & 46.629 & 46.353 \\
\hline 36 & 53.267 & 43.784 & 47.149 & 48.291 & 53.879 & 52.799 & 51.718 & 49.603 & 53.271 \\
\hline 37 & 37.017 & 37.417 & 38.723 & 38.307 & 40.899 & 39.049 & 37.198 & 38.599 & 37.018 \\
\hline 38 & 61.077 & 63.579 & 57.548 & 61.270 & 49.507 & 59.423 & 74.297 & 60.937 & 61.073 \\
\hline 39 & 15.973 & 15.838 & 15.892 & 16.140 & 15.990 & 16.420 & 16.850 & 16.188 & 15.974 \\
\hline 40 & 57.740 & 58.095 & 55.781 & 42.372 & 52.310 & 34.511 & 25.611 & 44.780 & 57.730 \\
\hline 41 & 54.060 & 50.327 & 49.561 & 54.300 & 48.283 & 58.273 & 73.259 & 55.667 & 54.058 \\
\hline 42 & 64.960 & 63.281 & 60.852 & 46.215 & 57.207 & 37.682 & 27.920 & 48.860 & 64.954 \\
\hline 43 & 17.610 & 17.285 & 17.575 & 17.794 & 18.107 & 18.262 & 18.417 & 17.907 & 17.609 \\
\hline 44 & 26.463 & 27.630 & 28.127 & 26.952 & 29.021 & 26.398 & 24.211 & 27.057 & 26.462 \\
\hline 45 & 13.534 & 13.429 & 13.539 & 13.705 & 13.714 & 13.938 & 14.186 & 13.752 & 13.533 \\
\hline 46 & 27.792 & 29.154 & 27.538 & 29.014 & 25.115 & 28.893 & 33.931 & 28.941 & 27.791 \\
\hline 47 & 57.207 & 56.498 & 60.252 & 54.925 & 67.761 & 53.667 & 44.270 & 56.229 & 57.201 \\
\hline 48 & 26.175 & 26.425 & 25.791 & 25.933 & 24.680 & 25.502 & 26.325 & 25.776 & 26.172 \\
\hline 49 & 29.164 & 25.751 & 28.168 & 27.855 & 33.607 & 29.958 & 27.039 & 28.730 & 29.171 \\
\hline 50 & 69.730 & 72.478 & 62.198 & 24.002 & 51.918 & 15.923 & 8.723 & 39.207 & 69.743 \\
\hline 51 & 27.351 & 27.038 & 23.104 & 27.794 & 30.502 & 28.550 & 26.988 & 28.163 & 27.351 \\
\hline 52 & 54.013 & 48.521 & 62.599 & 56.338 & 104.835 & 64.156 & 43.816 & 63.377 & 54.025 \\
\hline 53 & 23.940 & 25.793 & 24.273 & 23.403 & 22.145 & 21.730 & 21.314 & 23.110 & 23.942 \\
\hline 54 & 33.662 & 31.563 & 32.597 & 33.517 & 34.665 & 35.471 & 36.277 & 34.015 & 33.658 \\
\hline 55 & 60.995 & 65.333 & 66.003 & 63.928 & 67.009 & 62.874 & 58.739 & 63.981 & 60.992 \\
\hline 56 & 24.361 & 24.762 & 24.164 & 24.406 & 23.089 & 24.087 & 25.084 & 24.265 & 24.365 \\
\hline 57 & 65.621 & 63.235 & 61.902 & 46.895 & 59.904 & 38.725 & 28.135 & 49.799 & 65.619 \\
\hline 58 & 46.720 & 46.165 & 50.251 & 51.386 & 58.424 & 56.607 & 54.791 & 52.938 & 46.714 \\
\hline 59 & 67.386 & 78.648 & 75.403 & 73.655 & 70.535 & 69.911 & 69.287 & 72.907 & 67.388 \\
\hline 60 & 60.893 & 65.245 & 64.995 & 63.118 & 64.619 & 61.523 & 58.428 & 62.988 & 60.890 \\
\hline 61 & 51.344 & 45.209 & 48.518 & 49.717 & 55.136 & 54.225 & 53.313 & 51.020 & 51.343 \\
\hline 62 & 67.867 & 74.921 & 61.830 & 48.086 & 48.738 & 37.352 & 29.761 & 50.115 & 67.881 \\
\hline 63 & 39.419 & 36.202 & 37.819 & 38.862 & 41.052 & 41.523 & 41.994 & 39.575 & 39.422 \\
\hline 64 & 46.785 & 44.204 & 45.344 & 43.043 & 47.624 & 42.049 & 37.868 & 43.355 & 46.785 \\
\hline 65 & 53.728 & 51.270 & 49.062 & 51.798 & 46.118 & 52.220 & 61.373 & 51.974 & 53.726 \\
\hline 66 & 45.004 & 37.728 & 41.420 & 42.296 & 48.806 & 46.865 & 44.924 & 43.673 & 45.000 \\
\hline 67 & 48.864 & 51.153 & 51.663 & 50.041 & 52.428 & 49.207 & 45.987 & 50.080 & 48.864 \\
\hline 68 & 67.376 & 59.431 & 75.570 & 68.220 & 123.989 & 77.009 & 53.519 & 76.290 & 67.384 \\
\hline 69 & 43.068 & 42.070 & 40.205 & 40.707 & 37.874 & 39.733 & 42.212 & 40.467 & 43.069 \\
\hline 70 & 28.820 & 30.639 & 29.475 & 29.726 & 27.380 & 28.904 & 30.429 & 29.426 & 28.818 \\
\hline
\end{tabular}

\section{Discussion and conclusions}

A look at Table 1 clearly shows that the classical FDP method relying on the extrema positions works satisfactorily only for low velocity meteors while for medium and particularly high velocity meteors the agreement of $v_{i}$ with $\bar{v}$ is very poor. We can see that values of $\bar{v}$ can be both lower and higher than $v_{i}$ (e.g. cases numbers $2,3,6,22,26$, and many others). Also, nonhyperbolic meteors can become hyperbolic, e.g. cases nos. 13, 59, 68. This situation exists even though our generated FDP were not subject to any noise. Therefore, one cannot expect this method to work properly given the presence of noise in practical observations. Thus, the classical method of velocity determination making use of the extrema positions of the FDP should be rejected in practice. Moreover, it produces fictitious deceleration in the latter half of trails in most cases, and complete deceleration in case of meteors nos. 3,11, 28, and 53. Furthermore, if we consider only the sequence of velocities $v_{1-2}, v_{2-3}$, and $v_{3-4}$, we will infer deceleration for almost all cases. This indicates that the de- 


\begin{tabular}{|c|c|c|c|c|c|c|c|c|c|}
\hline \multicolumn{10}{|c|}{ The second continuation of Table 1.} \\
\hline$\overline{71}$ & 59.169 & $\overline{51.812}$ & 56.434 & 57.703 & 65.679 & $63 . \overline{94}$ & 61.509 & 59.455 & 59.161 \\
\hline 72 & 53.939 & 59.585 & 59.611 & 50.614 & 59.651 & 45.232 & 35.619 & 51.719 & 53.938 \\
\hline 73 & 36.558 & 32.208 & 33.722 & 34.647 & 36.749 & 37.085 & 37.422 & 35.305 & 36.556 \\
\hline 74 & 42.545 & 36.320 & 40.564 & 43.234 & 51.173 & 51.876 & 52.578 & 45.957 & 42.550 \\
\hline 75 & 46.855 & 38.299 & 43.065 & 45.876 & 54.980 & 55.348 & 55.715 & 48.880 & 46.857 \\
\hline 76 & 60.697 & 59.279 & 55.884 & 59.161 & 51.357 & 59.067 & 70.631 & 59.230 & 60.694 \\
\hline 77 & 38.881 & 39.892 & 38.230 & 38.404 & 35.321 & 37.101 & 38.882 & 37.972 & 38.877 \\
\hline 78 & 26.613 & 27.989 & 28.991 & 28.675 & 30.660 & 29.247 & 27.833 & 28.899 & 26.614 \\
\hline 79 & 23.544 & 23.544 & 24.530 & 23.996 & 26.108 & 24.358 & 22.609 & 24.191 & 23.541 \\
\hline 80 & 53.115 & 49.920 & 53.611 & 48.827 & 60.993 & 47.953 & 39.259 & 50.094 & 53.114 \\
\hline 81 & 24.517 & 24.452 & 24.592 & 24.830 & 24.842 & 25.169 & 25.497 & 24.897 & 24.515 \\
\hline 82 & 10.423 & 10.306 & 10.362 & 10.353 & 10.442 & 10.390 & 10.329 & 10.364 & 10.423 \\
\hline 83 & 40.564 & 34.848 & 39.728 & 38.037 & 51.928 & 41.225 & 34.090 & 39.976 & 40.571 \\
\hline 84 & 34.847 & 30.349 & 35.441 & 32.385 & 50.715 & 34.421 & 26.274 & 34.931 & 34.846 \\
\hline 85 & 39.026 & 41.685 & 42.165 & 38.377 & 42.964 & 36.014 & 30.802 & 38.668 & 39.030 \\
\hline 86 & 29.205 & 28.875 & 29.096 & 28.195 & 29.427 & 27.686 & 25.944 & 28.204 & 29.209 \\
\hline 87 & 30.229 & 29.172 & 29.937 & 30.787 & 31.468 & 32.401 & 33.335 & 31.183 & 30.227 \\
\hline 88 & 35.182 & 34.451 & 35.058 & 35.156 & 36.121 & 35.773 & 35.424 & 35.331 & 35.184 \\
\hline 89 & 67.852 & 68.071 & 66.995 & 65.198 & 65.383 & 63.043 & 60.703 & 64.899 & 67.846 \\
\hline 90 & 54.270 & 53.486 & 53.316 & 51.770 & 53.061 & 50.484 & 47.907 & 51.671 & 54.271 \\
\hline 91 & 12.481 & 12.555 & 12.583 & 12.315 & 12.631 & 12.112 & 11.645 & 12.307 & 12.481 \\
\hline 92 & 62.678 & 49.350 & 62.190 & 33.745 & 100.708 & 27.057 & 14.782 & 47.972 & 62.690 \\
\hline 93 & 37.831 & 44.140 & 42.063 & 39.999 & 39.294 & 37.239 & 35.183 & 39.653 & 37.831 \\
\hline 94 & 41.471 & 40.228 & 45.086 & 43.237 & 57.231 & 46.247 & 38.924 & 45.159 & 41.471 \\
\hline 95 & 55.742 & 49.326 & 56.120 & 56.917 & 69.709 & 64.508 & 59.307 & 59.314 & 55.739 \\
\hline 96 & 69.186 & 92.061 & 77.485 & 52.536 & 62.909 & 39.362 & 27.588 & 58.657 & 69.192 \\
\hline 97 & 56.438 & 51.448 & 55.931 & 57.206 & 64.899 & 62.964 & 61.029 & 58.913 & 56.442 \\
\hline 98 & 18.709 & 18.132 & 18.318 & 18.412 & 18.742 & 18.711 & 18.683 & 18.500 & 18.709 \\
\hline 99 & 20.159 & 20.558 & 20.192 & 19.867 & 19.618 & 19.325 & 19.033 & 19.766 & 20.164 \\
\hline 100 & 30.947 & 31.324 & 31.140 & 31.591 & 30.883 & 31.799 & 32.945 & 31.614 & 30.943 \\
\hline
\end{tabular}

celeration resulting from the application of the classical method is false, a result which can also be understood from the fact that the classical FDP has been derived under the assumption of constant meteoroid velocity. This could indicate that the decelerations following from the quasi-simple processing of diffraction pattern as reported by Baggaley et al. (1994) should be treated with care. On the other hand, the NLSF method yields quite satisfactory results as can easily be seen from Table 1 . Only the task of obtaining the standard deviation of the parameters it supplies remains open. This is beyond the scope of this work and will be treated in another article. This method is capable of yielding not only the velocity of the meteoroid, but also other parameters such as the time of passage of the specular point by the meteoroid and the ambipolar diffusion coefficient. The results are not biased by aliasing effects and it works quite properly within the whole velocity range considered even though we generated the FDP for a pulse repetition frequency of $379 \mathrm{~Hz}$. The situation of very high speeds where only a few extrema are present should also be investigated. The NLSF method appears to produce reliable results within the usual meteor speed range. It should be used for determination of the velocity of radio meteors, at least at the first approximation stage.

Acknowledgments. This work was supported under grant No 303104 of Grant Agency of Academy of Sciences of the Czech Republic.

\section{References}

Baggaley, W. J., Bennett R. G. T., Steel D. I., \& Taylor, A. D. 1994, Q. J. R. astr. Soc., 35, 293

Pecina, P. 1988, Bull. Astron. Inst, Czechosl., 39, 193

Šimek, M. 1968, Canad. J. Phys., 46, 1563 